PROCEEDINGS OF THE

AMERICAN MATHEMATICAL SOCIETY

Volume 88, Number 2, June 1983

\title{
UPPER SEMICONTINUOUS COLLECTIONS OF CONTINUA IN CLASS $W$
}

\author{
C. WAYNE PROCTOR ${ }^{1}$
}

\begin{abstract}
A continuum is proven to be in Class $W$ if it can be decomposed into an upper semicontinuous collection of $C$-sets, each of which is contained in Class $W$, and if the upper semicontinuous decomposition space thus formed is in Class $W$.
\end{abstract}

1. Introduction. Since A. Lelek [9] first introduced a collection of continua known as Class $W$, much attention has been given to the problem of determining which classes of continua (of course described in terms of various topological properties) are contained in Class $W$. D. R. Read [11] has proven that Class $W$ contains all chainable continua; G. A. Feuerbacher [2] has shown that nonplanar circle-like continua are all in Class $W ; \mathrm{H}$. Cook [1] has proven that all hereditarily indecomposable continua are in Class $W$; and, in a joint paper, J. Grispolakis and E. D. Tymchatyn [4] have shown that atriodic tree-like continua are in Class $W$. The main result of this paper is a theorem which is a generalization of Theorem 4.6 that appears in another paper by J. Grispolakis and E. D. Tymchatyn [3]. This result can be used in constructing continua in Class $W$, just as Theorem 3.4 in the Grispolakis and Tymchatyn paper [3] gives a construction for a large class of continua in Class $W$. An example of a continuum is given which is in Class $W$ but is not tree-like, not circle-like, nor hereditarily indecomposable.

2. Definitions. All continua are taken to be compact, connected, metric spaces. A mapping (continuous function) $f$ from a topological space $X$ onto a topological space $Y$ is weakly confluent if and only if for each continuum $K$ of $Y$ there is a component of $f^{-1}(K)$ that is mapped onto $K$ by $f$. A continuum $M$ is said to be in Class $W$ if and only if all mappings from continua onto $M$ are weakly confluent.

If $K$ is a subcontinuum of a continuum $M$, then $K$ is said to be a $C$-set in $M$ if and only if each subcontinuum of $M$ containing a point of $K$ is either a subset of $K$ or has $K$ as a subset.

If $\mathcal{G}$ is a collection of disjoint closed sets whose union is a continuum $M$, then $\mathcal{G}$ is said to be an upper semicontinuous collection if and only if for each $K$ in $\mathcal{S}$ and each open set $U$ in $M$ containing $K$ as a subset there is an open set $V$ in $M$ containing $K$ with the property that each closed set in $\mathcal{G}$ that intersects $V$ is also a subset of $U$. The upper semicontinuous decomposition space $\mathscr{D}$ formed by the upper semicontinuous

Received by the editors October 1, 1982 and, in revised form, November 18, 1982.

1980 Mathematics Subject Classification. Primary 54B15, 54F15; Secondary 54B25, 54C10.

${ }^{1}$ Research partially supported by a Stephen F. Austin State University Faculty Research Grant. 
collection $\mathcal{G}$ as described above has the property that the points of the space $\mathscr{D}$ are the elements contained in $\mathcal{G}$, and $\mathscr{U}$ is an open subset of $\mathscr{D}$ if and only if $\mathscr{U}$ is a subset of $\mathcal{G}$ such that the union of all of the elements in $\mathcal{G}$ contained in $\mathscr{U}$ forms an open subset of $M$. The natural mapping which is said to induce the upper semicontinuous decomposition space $\mathscr{D}$ is the mapping $\eta: M \rightarrow \mathscr{D}$ defined such that $\eta(x)$ is the element of $\mathcal{G}$ that contains $x$ for each $x$ in $M$.

\section{Main result.}

THEOREM. If $M$ is a continuum which can be decomposed into an upper semicontinuous collection $\mathcal{G}$ of $C$-sets, each of which is contained in Class $W$ such that the upper semicontinuous decomposition space formed by the collection $\mathcal{G}$ is also in Class $W$, then the continuum $M$ is in Class $W$.

Proof. Suppose $f$ is a mapping from an arbitrary continuum $X$ onto $M$. Let $K$ be a subcontinuum of $M$. The main objective is to show that some subcontinuum of $f^{-1}(K)$ is mapped onto $K$ by $f$. To accomplish this, two cases must be considered, i.e. whether $K$ is not a subset of any element of $\mathcal{G}$ or whether $K$ is a subset of some element of $\mathcal{G}$.

First, suppose $K$ is not a subset of any element of $\mathcal{G}$. Since each element of $\mathcal{G}$ is a $C$-set in $M$, the continuum $K$ is the union of all the elements of $\mathcal{G}$ which intersect $K$. Allowing $\eta: M \rightarrow D$ to represent the natural mapping that induces the upper semicontinuous decomposition space $\mathscr{D}$ formed by $\mathcal{G}$, the mapping $(\eta \circ f): X \rightarrow \mathscr{D}$ is weakly confluent since $\mathscr{D}$ is in Class $W$; thus there is a subcontinuum $C$ of $(\eta \circ f)^{-1}(\eta(K))$ that is mapped onto $\eta(K)$ by $\eta \circ f$. Now, notice that $f(C)$ is a continuum in $M$ that intersects each set in $\mathcal{G}$ that contains a point of $K$ due to the manner in which $\eta$ is defined. This shows that $K$ is a subset of $f(C)$ since each set in $\mathcal{G}$ that contains a point of $K$ is a subset of $f(C)$. Also, notice that each point of $f(C)$ is mapped by $\eta$ to an element of $\mathcal{G}$ that contains a point of $K$, but each element of $\mathcal{G}$ that contains a point of $K$ is a subset of $K$, which proves that each point of $f(C)$ is in $K$. This demonstrates that there is a subcontinuum, namely $C$, of $f^{-1}(K)$ that is mapped by $f$ onto $K$ if $K$ is not a subset of any element of $\mathcal{G}$.

Now, suppose that $K$ is a subset of some element $H$ of $\mathcal{G}$. W. T. Ingram [8] has proven that a $C$-set such as $H$ has the property that every component of $f^{-1}(H)$ is thrown by $f$ onto $H$. Let $C^{\prime}$ denote some component of $f^{-1}(H)$ that has $f\left(C^{\prime}\right)=H$. Now, using the fact that $H$ is in Class $W$ and that $f$ restricted to $C^{\prime}$ is a mapping from $C^{\prime}$ onto $H$, there is a subcontinuum of $C^{\prime}$ that is mapped by $f$ onto $K$. This concludes the proof of the theorem.

4. Example. An interesting example of a continuum within Class $W$ can be constructed by first letting $\left\{r_{1}, r_{2}, r_{3}, \ldots\right\}$ denote the set of all rationals within the open interval $(0,1)$. A continuum $X_{1}$ is defined to be the same as the closed interval $[0,1]$ except the rational $r_{1}$ is replaced with the dyadic solenoid (the inverse limit of the system which has the complex unit circle as each of its coordinate spaces and $f(Z)=Z^{2}$ as each of its bonding maps) in such a manner that the half-open intervals $\left[0, r_{1}\right)$ and $\left(r_{1}, 1\right]$ spiral down to the dyadic solenoid, making the solenoid a 
$C$-set in $X_{1}$. The spiraling down to the dyadic solenoid can be accomplished by first selecting a composant of the solenoid and then winding each of the rays $\left[0, r_{1}\right)$ and $\left(r_{1}, 1\right]$ back and forth over the composant, similar to the way the graph of $y=$ $x^{-1} \sin x^{-1}$ approaches the $y$-axis, such that the closures of each of the rays will contain the composant. Inductively, $X_{n+1}$ is defined to be the same as $X_{n}$ except $r_{n+1}$ is replaced with a topological copy of the dyadic solenoid in a way similar to that described above so that the solenoid being added is disjoint from the remaining two components of $X_{n}$ once $r_{n+1}$ is removed and so that each of the components spiral to the newly added dyadic solenoid. Now consider $f_{n}$ to be the mapping that maps $X_{n+1}$ onto $X_{n}$ such that $f_{n}$ maps to the point $r_{n+1}$ in $X_{n}$ each point of the solenoid in $X_{n+1}$ that replaced $r_{n+1}$ in the construction of $X_{n+1}$, and $f_{n}$ is the same as the identity map elsewhere on $X_{n+1}$. Letting $X_{\infty}$ denote the inverse limit of the system which has $X_{n}$ as its $n$th coordinate space and $f_{n}$ as its $n$th bonding map, it can be easily seen that $X_{\infty}$ can be decomposed into an upper semicontinuous collection $\mathcal{G}$ of $C$-sets such that $\mathcal{G}$ contains $Y$ if and only if for some natural number $n$ the $n$th projection of $Y$ into $X_{n}$ is the dyadic solenoid that was inserted in place of $r_{n}$ or $Y$ is a set consisting of a single point, none of whose coordinates belong to one of the inserted solenoids. As a result of G. A. Feuerbacher's work [2], each element of $\mathcal{G}$ is in Class $W$. It should also be noticed that the upper semicontinuous decomposition space $\mathscr{Q}$ formed by $\mathcal{G}$ is an arc which places $\mathscr{D}$ in Class $W$; thus, $X_{\infty}$ is in Class $W$ by applying the theorem. It should be observed that this example is neither tree-like, circle-like, nor hereditarily indecomposable.

\section{REFERENCES}

1. H. Cook, Continua which admit only the identity mapping onto non-degenerate subcontinua, Fund. Math. 60 (1967), 241-249.

2. G. A. Feuerbacher, Weakly chainable circle-like continua, Dissertation, Univ. of Houston, Houston, 1974.

3. J. Grispolakis and E. D. Tymchatyn, Continua which admit only certain classes of onto mappings, Topology Proc. 3 (1978), 347-382.

4. __ Continua which are images of weakly confluent mappings only, Houston J. Math. 5 (1979), $483-502$.

5. On confluent mappings and essential mappings - a survey, Rocky Mountain J. Math. 11 (1981), 131-153.

6. J. G. Hocking and G. S. Young, Topology, Addison-Wesley, Reading, Mass., 1961.

7. W. T. Ingram, Atriodic tree-like continua and the span of mappings, Topology Proc. 1 (1976), 329-333.

8. _ C-sets and mappings of continua, Topology Conf. (to appear).

9. A. Lelek, Report on weakly confluent mappings, (Topology Conf., Virginia Polytechnic Inst. and State Univ., 1973), Lecture Notes in Math., vol. 375, Springer, Berlin, 1974, pp. 168-170.

10. R. L. Moore, Foundatins of point set theory, Amer. Math. Soc. Colloq. Publ., vol. 13, Amer. Math. Soc., Providence, R.I., 1962.

11. D. R. Read, Confluent and related mapping, Colloq. Math. 29 (1974), 233-239.

12. A. D. Wallace, The position of C-sets in semigroups, Proc. Amer. Math. Soc. 6 (1955), 639-642.

Department of Mathematics, Stephen F. Austin State University, Nacogdoches, Texas 75962 